MUZIKOLOŠKI ZBORNIK - MUSICOLOGICAL ANNUAL IX, LJUBLJANA 1973

UDK $785.74: 787.1 / .4$ Beethoven

\title{
ZUM PROBLEM DER LETZTEN STREICHQUARTETTE BEETHOVENS
}

Wolfgang B oetticher (Göttingen)

Beethovens fünf späte Streichquartette bilden eine geschlossene Gruppe: op. 127 Es-Dur (1822-25), op. 130 B-Dur (1825-26), op. 131 cis-moll (Ende 1825-26), op. 132 a-moll (Ende 1824-25), op. 135 F-Dur (Sommer 1825), der ursprünglich für op. 130 bestimmte Finalsatz, die Fuge B-Dur (Herbst 1825), als op. 133 ediert, sowie der hierfür - auf Anregung des Verlegers M. Artaria - eintretende Finalsatz (September bis Anfang November 1826). Seit langem wird dieser Werkkreis als exemplarischer Fall für den zunächst problematischen Begriff eines »Spätwerks« eingeschätzt. Die Einheitlichkeit ist durch die Distanz zu allem Vorausgegangenem, der Sinfonik, der Klavierwerke (Sonate, Bagatelle, Variation), der Missa solemnis dokumentiert, um nur die quantitativ herausragenden Leistungen $\mathrm{zu}$ nennen. Die seit 1945 paläographisch gesicherte Rückdatierung des Rondo op. 129 auf 1795-1798 sei nur am Rande erwähnt; ${ }^{1}$ op. 136 ist Herbst 1814 entstanden, op. 137, die Fuge D-Dur für Streichquintett gehört nach autographer Datierung in den November 1817. Insofern ist tatsächlich mit den fünf Streichquartetten ein opus ultimum geboten, das hohe Isolation gegenüber jüngsten Gattungsbelegen verrät, wobei kleine Gelegenheitsleistungen in der Spätphase ästhetisch deutlich Abstand wahren. ${ }^{2}$ Merkwürdig, daß Beethoven noch Anfang November 1826 mit einem Streichquintett beginnen wollte, worauf schon

${ }^{1}$ O. E. Albrecht in Musical Quarterly XXXI, 1945, S. 495 und E. Hertzmann ibid. XXXII, 1946, S. $171 \mathrm{ff}$.

2 In die beiden letzten Schaffensjahre 1825 und 1826 fallen nur noch der Dedikationskanon "Gott ist eine feste Burg", "Doktor sperr das Tor dem Tod", »Ich war hier, Doktor", ein weiterer für den holländischen Maler de Boer, der kleine Notenscherz auf Carl Holz' Quartettspiel, "Tobias" 1825 und im Folgejahr zwei weitere Notenscherze für den Wiener Verleger Tobias Haslinger, der Leitspruch "Das Schöne...", "Kühl, nicht lau" (Anspielung auf F. Kuhlau), "Ars longa", "Si non per portas«, "Freu' dich des Lebens", "Es muß sein", "Da ist das Werk", "Wir irren allesamt", ein kleines Klavierstück für Sarah Burney Payne, ferner Walzer und Eccosaise. Einiges findet sich in Briefen eingestreut. 
Nottebohm $\mathrm{m}^{3}$ anhand eines gleichzeitig datierten Blattes zu dem nachgelieferten Finalsatz von op. 130 hingewiesen hat. Im Klavierarrangement A. Diabellis sind hiervon 24 Takte als »Beethovens letzter musikalischer Gedanke« 1838 erschienen. Möglicherweise handelt es sich jedoch um einen Werkplan, den Beethoven schon in einem Hinweis an Diabelli 1824 gemeint haben kann. Immerhin ist das Autograph in seiner Existenz durch den Katalog der Nachlaßversteigerung 1827 verbürgt. Seit mehr als einem Jahrhundert bleibt es verschollen. Nicht auszuschließen ist, daß Beethoven zuletzt noch das Korpus der Quartette durch ein Quintett zu erweitern gedachte, wobei aber dieses "Andante maestoso«, so, wie wir es nur aus dem kleinen Torso kennen, keineswegs die Stilhöhe der letzten Kammermusik hält und nicht einmal den letzten geschlossenen Satz, das Finale zu op. 130 mit seinem straff-knöchernen thematischen Ansatz erreicht, der im Schrifttum keineswegs einheitlich hoch eingeschätzt wird. Zweifellos hatte Beethoven mit dem cis-moll-Quartett op. 131 den Parnaß beschritten. Man wird die geniale Spätgruppe mit diesem Werk (beendet Juli 1826) abzuschließen haben und dabei auch die wohl gleichzeitige Übertragung der Fuge op. 133 zu Klavier zu vier Händen (op.134) als Sekundärleistung ausscheiden dürfen.

Ist die Werkgruppe damit nach oben und unten abgegrenzt und in ihrer Ganzheit bestätigt, so stellt sich die Frage nach den Merkmalen eines spezifischen Spätstils nicht einfach. Daß es einen Generationenbegriff in der Kunstgeschichte gebe, der sich an der Distanz Lehrer - Schüler orientiert und daß nicht die absolute Zahl der Lebensjahre, sondern das relative "Alter" das Ende einer geistigbiologischen Entfaltungskurve anzeige, ist schon von den älteren Kunstwissenschaft (Wilhelm Pinder) in die Debatte geworfen worden. Bei Beethovens genannter Werkgruppe findet man zunächst die - vielleicht durch seine Taubheit verschärfte - Vereinzelung des Klangapparats, die starre Reduktion auf das Quattrocinium mit einer im Prinzip einheitlichen Klangerzeugung (Streicher), durchaus Merkmale einer „Grenzsituation«. Dennoch wird man zwei Aspekte getrennt verfolgen müssen: die Spätphase eines beethovenischen Personalstils und die Spätphase der Klassik selbst. Denn auch die "Alterung" der Klassik fällt in die gleichen Jahre, verbunden mit einer krisenhaften Konfrontation mit der aufkeimenden Romantik (C.M. v. Weber) und - immerhin nur 2-3 Jahre darauf - mit einem Hervortreten eines gänzlich neuen Stilbewußtseins, wie es sich sporadisch sehr instruktiv in dem noch unveröffentlichten viersätzigen

${ }^{3}$ G. Nottebohm, Beethoveniana, Leipzig-Winterthur 1872, S. 80 f., ferner derselbe, Zweite Beethoveniana, herausgegeben von E. Mandyczewski, Leipzig 1887, S. 522 f., Kinsky-Halm, Das Werk L. v. Beethovens ...., München-Duisburg o. J. (1955), S. 508. In der Gesamt-Ausgabe fehlend. Im übrigen vergleiche W. Hess in: Neues Beethoven-Jahrbuch VII, 1937, S. $104 \mathrm{ff}$. , hier als Nr. 28 geführt, A. Bruero, Beethoven, Catalogo ...., Rom 1951, S. 407, Nr. 174, ferner G. Lange in: Wissenschaftliche Beilage zu dem XXV. Jahresbericht des Humboldt-Gymnasiums zu Berlin, Berlin 1900, S. 8 und 10. 
Klavierquartett des Heidelberger Jura-Studenten Robert Schumann ${ }^{4}$ zeigt, der 1828-1829 neben tradiertem Motivvorrat gänzlich neue Wege beschreitet. Die Konfrontation ist weiterhin perfekt durch das Auftreten des frühen Mendelssohn und Chopin, wobei man bedenken muß, daß sich der jünglinghafte Nocturne-Typus als lyrisches Stimmungsstück bei Chopin aus wenig älteren Vorlagen entfaltet hat, also noch in Beethovens letzte Phase fällt. Neben Beethovens Personalstil befindet sich mithin eine sehr scharfe zeitstilistische Zäsur 1824-1829. Die plötzliche Umstellung wird indirekt bestätigt durch das auffällige, geradezu autoritäre Nachwirken des späten Beethoven in der jungen Romantiker-Generation, nicht zuletzt bei Chopin. ${ }^{5}$ Die alternden, der Erscheinung Beethovens anfangs günstig geneigten Kritiker standen gleichzeitig der Spätphase fassungslos gegenüber, wobei nur auf Rochlitz verwiesen sei ${ }^{6}$ der sich mit dem »fast Ungenießbaren" und »absichtlich Widerstrebenden" nicht abfinden konnte.

Im folgenden sei, nachdem die biographischen und stilpsychologischen Probleme im Umriß für die Werkgruppe diskutiert sind, der Versuch unternommen, einzelne Stilprädikate herauszupräparieren. ${ }^{7}$ Es handelt sich um ein zentrales Anliegen des Beethovenverständnisses, zu dem unter anderem wertvolle Beiträge zur Skizzentechnik durch v. Hecker, ${ }^{8}$ eine nur summarische Formenübersicht Jokls, ${ }^{9}$ eine anregende Erwägung zum Durchführungsproblem Rosenmanns ${ }^{10}$ und weitergehende Darlegungen Manns ${ }^{11}$ erreichbar sind, abgesehen von den im folgenden genannten Einzelstudien.

1. Schon in seiner denkwürdigen Studie zum Scherzo-Thema beobachtete Becking in seinem Schlußabschnitt zur »Geschichte der

${ }^{4}$ Nachgewiesen durch den Verfasser in: R. Schumann, Einführung in Persönlichkeit und Werk .... Berlin 1941, S. 640. Das Autograph befindet sich in Privatbesitz (Sammlung Wiede) und läßt bestimmte spezifisch romantische Ausdrucksmerkmale klar erkennen, die sodann in den frühen Klavierwerken Schumanns (op. 2, 4, 6 etc.) verwertet worden sind. Hierzu vergleiche auch H.F. Redlich, Schumann discoveries, in: Monthly Musical Record LXXX/LXXXI, London 1950/51, S. 134 ff., 182 ff., $261 \mathrm{ff.}$

5 W. Boetticher, Über einige Spätstilprobleme bei F. Chopin, in: Kongreß-Bericht F. Chopin (Warschau 16.-22. Februar 1960), Warschau 1963, S. $104 \mathrm{ff}$.

${ }^{6}$ H. Ehinger, Friedrich Rochlitz als Musikschriftsteller, Leipzig 1929 (= Sammlung Musikwissenschaftlicher Einzeldarstellungen, Heft IX), S. 77.

7 Zitiert nach Gesamt-Ausgabe, Serie VI, Band 2, S. 47-228.

8 J.v. Hecker, Untersuchungen an den Skizzen von Streichquartett op. 131 von Beethoven, Dissertation maschinenschriftlich Freiburg/Breisgau 1956.

9 E.Jokl, Die letzten Streichquartette Beethovens, Dissertation maschinenschriftlich Wien 1905.

10 M. Rosenmann, Studie zum Gestaltungsproblem der letzten fünf Streichquartette L. v. Beethovens, Dissertation maschinenschriftlich Wien 1930.

11 C.-H. Mann, Formale Probleme in den späten Werken Beethovens. Untersuchungen zum Stil der Kammermusik und des Klavierwerks, Dissertation maschinenschriftlich Hamburg 1955. 
Scherzowirkung «, ${ }^{2}$ daß, sofern wir - wie er schreibt - »echte Scherzi« von op. 130, 131, 135 im Auge behalten, der Verlauf "schnell« und "knapp " bis zum Extrem gesteigert ist,"3 was sich schon in der Klaviersonate op. 110 vorbereitet. Der Richtungsgegensatz der Themenhälften verhärtet sich, wobei bestimmte Merkmale, wie zum Beispiel die »bis zur Septe hinauflaufenden staccati«, wie Becking richtig bemerkt, ${ }^{14}$ quer durch Beethovens Schaffen nachweisbar bleiben, also unspezifisch sind. Zunächst aber fällt auf, daß der späte Beethoven der Benennung "Scherzo" fast konsequent ausweicht und auch den älteren Menuetttypus gänzlich eliminiert. Die radikale Simplifizierung und Verengung des scherzosen Themas kündigt sich schon in op. 106 (Hammerklavier-Sonate) an, steigert sich in op. 110 und erfährt sodann noch eine Weiterführung im Quartettbereich. Der kurzatmige Stoßcharakter vertieft sich noch durch vulgarisierende Rückleitungen unmittelbar vor der Themenreprise: in op. 130, Satz II am Ende des mittleren Trios ist es die dreifach gestaffelte, chromatische absteigende Linie in Viol. I (L'istesso tempo), wobei übrigens der vorausgehende diatonische Anstieg bereits in Satz I, Takt 50-52 (hier chromatisch) als sporadische Eröffnungsfigur einer neuen Themengruppe geistig fein vorbereitet ist. Das »Presto« in op. 131 verschärft als spätester Beleg den eng rotierenden Motivcharakter des Scherzos: hier erscheint gegenüber der Terzraumumspielung von op. 130 eine schematische Dreiklangsfigur, wobei die spitze Tonrepetition von op. 130 nun nicht etwa dem Trio (Viol. II, Viola, sodann die drei Unterstimmen) zugewiesen ist, sondern dem Phrasenende des Primärthemas selbst. Die Trios beider Werke verengen auffällig die Harmonik (wobei der bezweckte ältere musette-Effekt nebensächlich ist). Gegenüber dem noch punktierten "Scherzando vivace" in op. 127, des ältesten Belegs in der Werkgruppe, ist nun die glatte Rundläufigkeit unverkennbar. Der punktierte Duktus wird später außerhalb des Scherzo-Charakters nur noch im Marsch-Typus realisiert (op.132, Satz IV, „Alla Marcia, assai vivace«), der übrigens durch einen ähnlichen Vulgarisierungs-Prozeß (abgehackte Endungen der Melodiephrasen) belastet wird, sodaß fast ein grotesker, ironisierender Eindruck entsteht. Wie fein aber Beethoven bei solchen extremen Ausdrucksmitteln die Gesamtform abstimmend und kompensierend im Auge behielt, bezeugt der ungewöhnlich flache, fast bis zur Monotonie die Motivanteile wiederholende Pseudo-Scherzosatz des gleichen op. 132 (»Allegro ma non tanto«), dessen Trio mit den erwähnten Musette-Liegenoten klar den Satz als Stellvertreter für das herkömmliche Scherzo ausweist. Die bilaterale Anlage des Themas verleiht diesem Satz etwas diagrammhaftes: es ist die permanente Pendelführung in Frage und Antwort oder Systole und Dia-

${ }^{12}$ G. Becking, Studien zu Beethoven's Personalstil. Das Scherzothema, Leipzig 1921 (= Abhandlungen der Sächsischen Staatlichen Forschungsinstitute zu Leipzig.... Heft II), S. 77-147.

13 ibid., S. 143 f.

14 ibid., S. 146. 
stole, wobei die zweite Hälfte mehrfach wiederholt, auch verkürzt oder alleinstehend: Jd $_{\perp}$ eine eigentümliche Neutralität und aschfahle Färbung abgibt (trotz des szintillierenden Effekts im Trio). Zusammenfassend wird man als gänzlich neue Funktion des Scherzos festhalten können: Neigung zu kurzhebiger Stoßthematik, verbunden mit neutralisierenden, von einem Prozeß der "Intellektualisierung « diktierten, einförmigen Pendelreihen. Das erste Merkmal trifft für den raschen Typus zu, das zweite für den künstlich verlangsamten, nivellierenden Typus. Wenn man - allen methodischen Bedenken einer »Ưbertragbarkeit« solcher Merkmale zu Trotz - an einen Vergleich von Musik und Dichtung denken darf, dann wäre bezüglich des kurzhebigen Scherzo an den alten Goethe zu erinnern, der in der Spätfassung seines "Faust " auf "Kürze" achtete und ein »Hinwerfen der Worte« wagte: "Worte die wahren, Äther im Klaren; Ewigen Scharen, Überall Tag«. In solchem Zusammenhang steht auch Beethovens starre Unisono-Führung, die an vielen Orten über das gewohnte Maß hinausragt: im erwähnten Trio (op.132) am Schluß mit jener weit klaffenden Intervall-Breite, die nicht zuletzt auch die Quartettfuge op. 133 auszeichnet, wobei der Triller (bisher im Klavierwerk seit der Waldsteinsonate op. 53 bis zu den berühmten Ketten im Finalteil von op. 109 und 111 einen hellfunkelnden Orgelpunkt suggerierend) eine neue, vergröbernde Funktion gemeinsam mit einem gewaltsamen Unisono übernimmt. Solche harten Triller sind schon am Ende von op. $106 \mathrm{zu}$ beobachten und führen über den Klavierklang drastisch hinaus. Die sanft höherwindende, überleitende Wirkung einer Trillerkette ist zwar in den späten Quartetten beibehalten (op. 131, Satz IV, letzte Variation), scheint aber zugunsten härterer Mechanismen zurückzutreten. Am deutlichsten bezeugt dies die Unisono-Finalstrecke des nachgelieferten Schlußsatzes zu op.130, die eine harte Verdichtung bezweckt und jeder sanft aufsteigenden Vibration zuwiderläuft.

2. Ein weiterer Fragenkomplex ist die Abschwächung eines »spezifischen Klangs«. Schon die eingangs bemerkte "Reduktion« bis zum vierstimmigen Satz gleichartiger Instrumente spricht hierfür. Aber es sind weitere Merkmale zu erkennen, die sich zum Teil scheinbar aufheben, im Prinzip aber doch bestätigen. Evident ist die extrem hohe Führung der beiden Violinen, die fast wie eine Piccolo-Flöte klingen (zum Beispiel op. 130, Satz I, Takt 30-33, 35-36, 85-90 etc.). Hierzu gehört auch der statische, schwirrende Klangkomplex, der gelegentlich tatsächlich fast »stehend" bei enger Sekundumspielung auftritt (op. 131, Finalsatz, Takt 148-159), wobei die Achtelbewegung nicht als Abwandlung des Trillers mißverstanden werden sollte, im genannten Beispiel führt der Baß die Sekundschwankung abweichend in der augmentatio duplex und simplex (Halbe und Viertel) aus. Im weiteren Sinn sehen wir das Stilmerkmal in der "Klangperforation", wie sie am deutlichsten am Ende von op. 130, Satz III in den vibrierenden 32stel-Ketten zu Tage tritt. Diese durchweg feststellbare "Evaporierung" des Klangs schließt 
nicht aus, daß Beethoven an bestimmten Orten eine künstliche Umkehrung des Prinzips bezweckt, was zugleich ein Licht auf das Variations-Problem des letzten Beethoven wirft. Es handelt sich um eine forcierte Massierung des Klangs, die nur streckenweise auftritt und die nur als bewußte Ausnahme von der Grundregel einer Perforation des Klangs verstanden werden kann. Schon in der Klaviersonate op. 111, Satz II fällt vor Eintritt in die Schlußphase der Variationenkette eine geradezu chorhafte, »oratoriale "Massierung des Klangs auf, die über die Mittel des Klaviers hinausgeht. In op.131, Satz IV ist das letzte Glied der Variationen bis zur Persiflierung klanglich überladen: die Oberstimme ist durch Punktierung und Triller dick aufgetragen, die Mittelstimmen sind bereits vierstimmig geführt, das Cello bewegt sich in abgekürzten Alberti-Bässen mit ungelenken Impulsen. Durch grobe Verdopplung der Melodietöne im Oktavabstand (die mit den Trillern eine »Unreinheit" der Intonation erzeugen) ist hier eine wohlberechnete "Negation" des genannten Prinzips einer Evakuation und Spaltung der Klangelemente geschaffen. Das Auseinander-Legen der verdünnten Klangzonen bis zu zwei Extremen mit vielen Oktaven Abstand bereitet sich übrigens in op. 111 vor (Satz II). Eine weitere Verflüchtigung ist mit der stretta-Strecke in op. 131, Satz V (»sul ponticello«) geboten, die Mitwirkung der pizzicati, die hier vorbereiten, ist nicht unbezeichnend.

3. Die »chloralartige« Themenführung ist wohl das sinnfälligste Merkmal der letzten Quartette. Auch sie ist streckenweise auf bewußte Negationen und Perversionen angewiesen, wobei man nicht übersehen darf, daß Beethoven zuletzt durchweg auf eine quadratische Gesamtform des Quartetts in seiner Satzfolge bedacht war und, was für die Choralthemen wichtig ist, eigentlich keine Ausnahme zuließ. ${ }^{15}$ Der im älteren und jüngeren Schrifttum keineswegs einhellig anerkannte Satz III von op. $130,{ }^{16}$ "poco scherzoso" vorgeschrieben, ist ein Beispiel für diese »Kompensationstechnik« Beethovens. Er entwirft hier ein »Andante con moto ma non troppo in sehr breitem Rahmen, das - trotz des widersinnig pathetischen Vorhalts im Eröffnungstakt - nirgends die Thematik voll ausströ-

15 Beethoven ist keineswegs - wie im Schrifttum mehrmals eingeräumt wird - in op. 135 zum viersätzigen Schema »wieder zurückgekehrt«, was ohnehin der Zeitfolge der letzten Quartette widerspräche. Tatsächlich ist auch in op. 131 das quadratische Schema einer Satzfolge streng geboten, wenn man Satz I als Praeludium zu Satz II versteht, Satz III als Úberleitung zu Satz IV fixiert und Satz V die Stellvertretung eines einzelstehenden älteren »Scherzo« einräumt. Dann erscheint Satz VI sinnvoll als Zwischenposition zum Finale Satz VII. Die überleitende Funktion des Satzes VI wird zugleich genutzt, um in der zweiten Themenhälfte durch diatonischen punktierten Abstieg eine Rückerinnerung zum Satz I zu schaffen, was dem Ganzen seinen Zusammenhalt verleiht. Das dabei auftretende »Deszendenzmelos « hängt zugleicht mit dem im folgenden erörterten »alten Stil« bei Beethoven zusammen.

16 Hierzu jüngst unter anderem D. W. MacArdle, Analisi del quartetto op. 130 di Beethoven (englisch), in: Music Review, London 1947, Februar-Heft. 
men läßt, ja vorzeitig abricht, in pizzicati auflöst oder entstellt. Ein Eindruck, der sich durch die schlendernde staccato-Führung des Cello noch vertieft. Die Ambivalenz dieses Satzes steigert sich bis zur bewußten Irreführung: neben dem erwähnten pathetischen Vorhalt, der wiederholt auftritt, erscheint eine weitausholende Kadenz (»non troppo presto«) bei Reprisenbeginn, die eigentlich ins Nichts führt (Takt 24 vor dem Schluß). Lediglich in der kurzen Finalstrecke Takt 15-14 vor dem Ende des Satzes ist das Motiv in sehnsüchtigem Schweben, ganz frei von dem negierenden Grundwert, als ein kleiner Lichtblick dargestellt. ${ }^{17}$ Solche Negation einer choralartigen Themenkonzeption ist in op. 130 nur verständlich, wenn man die folgende "Cavatina", als den eigentlichen Ruhepunkt des Ganzen begreift. Aber selbst dieser viel bewunderte Satz enthält in der Mitte eine drastische Negation mit der kurzen Strecke "beklemmt" (die sich täuschend ähnlich schon in der "zerfranzten" Reprise des Arioso dolente der Klaviersonate op. 110 und auch in ihrer vorangehenden Akkord-Repetition ankündigt). Dieser »anti-choralische« Effekt dient nur dem primären Ausdrucksziel, einer getragen-feierlichen melodischen Physiognomie als letztem Thementypus Beethovens schlechthin im Andante oder Adagio. Bei der Cavatina ist konstituierend der Initialsprung der aufsteigenden Sexte, in geistiger Nachbarschaft zu der fallenden Quarte des Variationenthemas in op.111, Satz II. Die plastische Pfeiler-Wirkung eines solchen Intervalls ermöglicht es Beethoven, die Melodik in vielfach verschlungenem Strömen $\mathrm{zu}$ entfalten und ihren Vortrag fast in Zeitlupe $\mathrm{zu}$ zerdehnen. Ähnlich die Aufgabe der eröffnenden Sexte im »Heiligen Dankgesang...« von op.132, hier in polyphoner Staffelung, aber mit den gleichen, durch Synkopierung eintretenden, "zerfranzten« Gegenwirkungen zum primären choralartigen Klangfluß (Takt 85 ff. etc.). Beethoven hat diese sonderbare Technik vielfacher Windung und nicht erlahmender Fortspinnung seriöser Melodietypen zuletzt offenkundig als ein besonderes Ausdrucksproblem verfolgt. Im »Heiligen Dankgesang... แ bedient er sich diastematischer Mittel (künstliche Umkehrung des Initialmotivs etc.), vor allem aber gehört in diesen Stilkreis die hier gewählte Sonderart des »Lydischen«. Diese archaische Tonart sollte weniger emotional verstanden werden, sondern als Mittel, den schwebenden Charakter der Melodik zu unterstützen (das Lydische wird - ins neuere Dur-System projiziert als Doppelexistenz von F-Dur und C-Dur verstanden und läßt den Hörer, der psychologisch im neueren Quintzirkel denkt, mehrmals im Unklaren). Es scheint, als habe der Schlußsatz des gleichen Quartetts eine ähnliche Irritierung des Hörers bezweckt, wobei das gleiche "Schwebephänomen«, sei es melodisch oder tonal, vermutet

${ }^{17}$ Diese Stelle ist im Schrifttum seit langem Gegenstand der Bewunderung, ohne daß ihr eigentlich der rechte Stellenwert im Umkreis eines sunerfüllten" Satzes zuerkannt worden ist. Es sei vor allem auf A.W. Thayer, L. v. Beethovens Leben, Band V, weitergeführt von H. Deiters, herausgegeben von H. Riemann, Leipzig 1908, S. 293 verwiesen. 
werden muß. Das Hauptthema dieses Finalsatzes (Rondò) fixiert sich in seiner Mitte unerwartet auf der 4. Stufe, die zunächst als Dominante der Durparallele verstanden wird. Erst in der zweiten Themenhälfte wird sie als Subdominante der Haupttonart (Moll) erkannt und führt damit logisch zum Melodieziel. Dieser anti-dominantische Effekt in Themenmitte ist auch der choralähnlichen Nr. VI in op. 131 (Takt 10 etc.) eigen. Das anschaulichste Beispiel dieses feierlich-zeremoniellen Thementypus bietet der I. Satz von op. 131, der schon in seiner Tonartencharakteristik (cis-moll) auffällt. Er ist im "stile antico" entworfen, was für sich aber noch kein echtes Merkmal des späten Beethoven sein kann, denn sein Werk durchzieht in allen Phasen eine Adaption historischer Stile, worauf jüngst wieder $\mathrm{Kirby}^{18}$ aufmerksam gemacht hat. Allerdings darf man bestimmte »einschneidende" Tonschritte, wie zum Beispiel den Quartfall als barockes Stilmerkmal ${ }^{19}$ als eine Sonderart der Spätwerke Beethovens erkennen. Ist die intervallische Tonsprache zunehmend "prägnant«, so bleibt - und dies begünstigt die choralartige Anlage vieler Hauptthemen - der Satzbeginn vielfach neutral und unspezifisch. Mit Recht formuliert Müller-Blattau ${ }^{20}$ als Indiz der späten Quartettgruppe: „Wir vermissen die kraftvolle und eindeutige Prägung des Anfangens« gegenüber der gewohnten Sinfonik der mittleren Schaffensphase. Satz I von op. 131 ist in diesem Sinn tatsächlich auf einem impulsfreien Thema basierend. Es liegt eine antikisierende Anlehnung an das vokale Thema (besser: "soggetto" im Sinne der zeitgenössischen Terminologie) des späten 16. Jahrhunderts vor, die Technik nähert sich dem älteren Ricercare, allerdings mit der "modernen" Zutat des schwellenden $<\mathrm{sf}>\mathrm{p}$, das spätklassischer Sinfonik entstammt und der älteren Dynamik widerspricht. Überhaupt darf dieser Satz als großartige Synthese »alten" und "neuen" Stils gelten, da der vokalische Thementypus, um einen Zentralton kreisend, durchaus antiquirt ist, seine Fortspinnung aber sich, einer sprengenden, sequenzierenden Steigerung bedient, die sinfonische Herkunft verrät (Takt 57-61). Der choralartige Melodietypus hängt aber - in seiner Fortspinnung - mit der komplementären Rhythmik zusammen, die, wie Forchert ${ }^{21}$ jüngst richtig beobachtet, in der Geschichte des Streichquartetts bei dem späten Beethoven neue Akzente setzt: die Autorität der Einzelstimme wird abgeschwächt zugunsten eines korporativen Verständnisses des vier-

18 F. E. Kirby, Beethovens Gebrauch von charakteristischen Stilen. Ein Beitrag zur Einheit in der Mehrsätzigkeit, in: Kongreß-Bericht Gesellschaft für Musikforschung Bonn 1970, Kassel (Bärenreiter) s. a. (1972), S. $452 \mathrm{ff}$

19 E. Schenk, Barock bei Beethoven, in: Festschrift Ludwig Schiedermaier, Berlin-Bonn 1937, S. 177 ff.

20 J. Müller-Blattau, Beethoven im Spätwerk, in: Festschrift Max Schneider zum 80. Geburtstag, Leipzig 1955, S. 220.

21 A. Forchert, Rhythmische Probleme in Beethovens späten Streichquartetten, in: Kongreß-Bericht Gesellschaft für Musikforschung a. a.O., S. $394 \mathrm{f}$. 
stimmigen Satzes, was - über interne Form- und Klangprobleme hinaus - auch gesellschaftliche Konsequenzen für die Spieler und Hörer hat. Was wir von den Skizzierungen dieses I. Satzes von op. 131 aus verschiedenen Quellen kennen, hat schon v. Hecker ${ }^{22}$ zur Skepsis bewogen, daß solche fragmentarischen Entwürfte kaum die Genesis des Satzes aufhellen und »ein nicht immer lösbares Problem" darstellen. Ähnliche Vorbehalte zur Stilerkenntnis äußert jüngst Stadlen, ${ }^{23}$ aber schon Nottebohm ${ }^{24}$ bemerkte kritisch den "Eindruck einer Improvisation « in Bezug auf den Satz I von op. 131 in den Frühfassungen. Immerhin scheint die »Vokalität« des Thementypus als Spezifikum des späten Beethoven hier auch in den Entwürfen von Anfang an festzustehen und sie erhält damit - schon von Bauer ${ }^{25}$ beiläufig erkannt - schärfere Belichtung. Scheidet man einmal die Skizzen als Dokumentation aus, so verbleibt als wichtigstes Indiz die schon seit P. Bekker beschriebene Neigung zu elastischen Urthemen, ${ }^{26}$ die der eigentliche Mutterboden der choralartigen Themen darstellen. Dies trifft sich mit der Tendenz des späten Beethoven, die übliche Disposition eines Sonatensatzes (Exposition - Durchführung - Reprise) abzubauen und ihr einen Rondocharakter, also eine alternierende Form, aufzunötigen. In einem abweichenden $\mathrm{Zu}$ sammenhang, nämlich im Umkreis der Finalsätze, hat jüngst Stephan $^{27}$ auf die Infiltration des Rondo aufmerksam gemacht, was sich auch durch die Analysen der Finalfuge, die Deutsch ${ }^{28}$ vorgelegt hat, zu bestätigen schneint. In unserem Fall eines Eröffnungssatzes von op. 131 als Pseudo-Ricercare liegen die Dinge ähnlich: es tauchen couplet-ähnliche Zwischenstrecken auf, vor allem wird das dialektische Prinzip der Durchführung in der Mitte, das zentrale Anliegen der Hochklassik, abgeschwächt, sodaß sich der Satz in der Tat der monothematischen Anlage der späteren Romantik nähert. Auch die Reprise erscheint beeinträchtigt und weicht freieren Coda-Gruppen. Die Abhängigkeit dieser großformalen Verschiebung von dem Thema selbst ist evident. Es ist vielfach auf diesen feierlichen Thementypus

${ }^{22}$ a. a. O., S. 194. Hierzu vergleiche auch H. Unverricht, Skizze - Brouillon - Endfassung. Definitions- und Bestimmungsschwierigkeiten bei den Skizzen Beethovens, in: Kongreß-Bericht Gesellschaft für Musikforschung a. a. O., S. 99 .

${ }_{23}^{2}$ P. Stadlen, Possibilities of an aesthetic evaluation of Beethoven's sketches, in: Kongreß-Bericht Gesellschaft für Musikforschung, a.a. O., S. $111 \mathrm{ff}$.

24 G. Nottenbohm, Beethoveniana, a. a. O., S. 54-59.

${ }^{25}$ M. Bauer, Formprobleme des späten Beethoven, in: Zeitschrift für Musikwissenschaft IX, 1926/1927, S. 341.

${ }^{26}$ Es sei unter dem jüngeren Schrifttum zu diesem Problem auf die trefflichen Studien von D. Cooke, The unity of Beethoven's late Quartets, in: Music Review, London 1963, S. 30 ff. und von J. Kerman, The Beethoven Quartets, London 1967, verwiesen.

${ }_{27}$ R. Stephan, $\mathrm{Zu}$ Beethovens letzten Quartetten, in: Die Musikforschung XXIII, 1970, S. 249, $254 \mathrm{f}$.

${ }^{28} \mathrm{~F}$. Deutsch, Die Fugenarbeit in den Werken Beethovens, in: Beethoven-Zentenarfeier, Festschrift, Wien 1927, S. 103. 
verwiesen worden,29 aber seine eigentliche Konsequenz für die Gestalt des ganzen Satzbildes sei hier ergänzend bemerkt. Man wird dabei einen gewissen Akzent zwischen Eröffnungssatz und Finalsatz sehen müssen, denn Beethoven scheint zuletzt nicht gleichartig die zugehörigen Themen konzipiert zu haben. Neigt der Eröffnungssatz zum archaischen Stil, den Schmitz ${ }^{30}$ in anderem Zusammenhang als "Ablösung des obligaten Akkompagnements « durch eine "reine und eigene Polyphonie« im Spätwerk erkannt hat, so wendet sich der Schlußsatz eher jüngster Entwicklung $\mathrm{zu}$ und schafft manch lehrreiche Analogie zu Schubert, was schon Misch ${ }^{31}$ aufgefallen ist. Gemeinsam aber ist der verengend wirkende Terzraum, der sanft schwingend durchmessen wird. Seine konstituierende Bedeutung erkennt man zum Beispiel im Vergleich von op. 131, Satz I, Takt 1-4 mit Satz IV, Variation 4 und op. 132, Satz I, Seitenthema Takt 11-12. Aber diese enggewundene Vokalität kann nur als Sonderart des späten Beethoven richtig eingeschätzt werden, wenn man die zuvor beschriebene Neigung zu »klaffenden" Tonschritten im Thema (am deutlichsten in der Quartettfuge op. 133 und am Eingang von op.132, Satz I) als anderes Extrem gleichermaßen festhält. Wenn abermals ein Vergleich mit dem Spätwerk des Dichters gewagt werden darf, dann wäre zu nennen: die Häufung umschreibender Ausdrücke, das zähe Festhalten am Grundriß, die besondere Nachdrücklichkeit einzelner Stellen, die aber als Kraftakte von langer Hand vorbereitet sind, die geschraubten Einschübe, aber zugleich die strenge ökonomie, die sich auf das Wesentliche beschränkt. Tatsächlich sehen wir im Satz I von op. 131 jene im poetischen Alterswerk des öfteren erkannte Sonderleistung weiträumiger Praedisposition: der Satz erscheint als eine stabile Steigerung bis zum letzten Themeneinsatz im Cello Takt 99, der als resolutio des Ganzen (einziger unverkürzter Themenvortrag in der Baßregion, gesteigert durch Augmentation) verstanden sein will. Solch weiträumige Progression ist das eigentliche Anliegen Beethovens, die schwebende Vokalthematik nur die Voraussetzung. Es ist eine "Enttypisierung" und "Relativierung" des Themenverlaufs, die Beethovens eigener, früherer Praxis einer »thematischen Arbeit" deutlich widerspricht. Ein Zug ins "Generelle», "Entpersönlichende«, so wie es der Spätfassung von Goethes Faust zugrunde zu liegen scheint, da nur noch von einer Büßerin, „sonst Gretchen genannt «, die Rede ist. Man bedenke ferner, daß der fragliche Satz nur als Introduktion zum folgenden Allegro auftritt, mit-

${ }^{29}$ Es sei nur aus dem jüngeren Schrifttum auf die trefflichen Arbeiten von J.N. MacKee, Beethoven's last Quartets, in Musical Opinion LXXXII, 1958/1959, S. 377 ff. (mit Bezug auf D. G. Mason, The Quartets of Beethoven, New York 1948) und R. Fiske, Beethoven's last Quartets, London (Oxford University Press) 1940, 3. Auflage ibid. 1948 (= The Musical Pilgrim), S. 67 ff., verwiesen.

30 A. Schmitz, Beethoven, Bonn 1927, S. 149.

${ }^{31}$ L. Misch, Zwei B-Dur-Themen. Eine Beethoven-Schubert-Studie, in: L. Misch, Beethoven-Studien, Berlin 1950, S. 24 ff. (zuerst erschienen in: Acta Musicol. XIII, Kopenhagen 1941, S. 85 ff.). 
hin eine eigensubstanzliche Existenz verneint. Nur durch diese immanent-musikalischen Bezüge erhellt sich das Problem dieses Satzes und es zeigt sich, wie verfehlt die Resultate einer älteren Beethoven-Hermeneutik ${ }^{32}$ gewesen sind.

4. Neu sind auch die wiegenden, gelösten Themen in Nachbarschaft des vorerwähnten Ricercare-Typus, die sich dem Tanz nähern. Es sei auf op. 130, Satz IV "Alla danza tedesca" (Allegro assai) verwiesen, zu dem Misch ${ }^{33}$ eine feinsinnige Studie und Romain Rolland ${ }^{34}$ eine poetische Imagination beigetragen haben. Die ältere Literatur, so $\mathrm{Helm}^{35}$ erkannte nur einen »anspruchslosen « Tanzsatz mit einem Trio »derber Landlust«, während neuerdings Mason ${ }^{36}$ im Walzerthema eher eine Retrospektive in die Jugendzeit vermutet. Die Einschätzung ist mithin keineswegs einheitlich, abgesehen von der umstrittenen Zugehörigkeit des Satzes zu op. 130.37 Was den Spätstil Beethovens betrifft, so sehen wir zunächt die bisher ungewohnte, versteifende, »axiale« Wiegebewegung im Dreiklangsmotiv. Die volltaktige Bildung im 3/8 repräsentiert den seriösen Ländler jüngster Prägung. Hier traf Beethoven eine subjektive Wahl, denn gleichzeitig war, wie Carner $^{38}$ nachgewiesen hat, der beschleunigende Wiener »Geschwindwalzer" verbreitet. Beethoven suchte demgegenüber eine enge Fesselung der verlangsamten Figur, was auch der Nachsatz Takt 25 ff. bestätigt. Die dörflich-rustikalen gestoßenen Achtel in den drei Begleitinstrumenten Takt 89-96 und 106-112 sollte man nicht so sehr als vulgarisierende Vorstadtmusik, wie im bisherigen Schrifttum, begreifen. Wichtiger erscheint die starre Fixation, die erzwungene, stereotype Begleitfunktion. Der Satzschluß aber verweist uns erneut auf die "Perforierung" des Klanges: $a b$ Takt 129 wird das Thema dialogisch aufgelöst und - als eine riesige Penultima - in ein falsches tonales Gleis (Subdominante) abgezweigt, was neben der Verdünnung des Klangs eine erhebliche Irritierung bedeutet.

5. Wichtig ist die neue Rolle des Recitativo. Es tritt am deutlichsten in op. 131, Satz III (Allegro moderato, Più vivace) und op. 132,

32 Thayer-Deiters-Riemann a. a. O., Band V, 5. Auflage 1911, S.320: "... den von der Welt abgekehrten, ganz innerlich lebenden Meister..., mit dem Drucke, der Krankheit und seelische Leiden auf ihn gelegt, der sich durch Erinnerung, Wünsche, Hoffnungen denselben $\mathrm{zu}$ entziehen sucht ...."

33 L. Misch, Alla danza tedesca, in: L. Misch, Beethoven-Studien a. .a. O., S. 20 ff. (zuerst erschienen in: Allgemeine Musik-Zeitung 1934, Nr. 34).

${ }_{34}$ R. Rolland, Les derniers Quatuors, in: R. Rolland, Beethoven, les grandes époques créatrices, Band V, Paris 1948, S. $36 \mathrm{ff}$.

${ }^{35}$ Th. Helm, Beethoven's Streichquartette, Versuch einer technischen Analyse ..., 2. Auflage Leipzig 1910, S. 245 f.

${ }_{36}$ D. G. Mason, The Quartets of Beethoven, New York (Oxford University Press) 1947, hier zitiert nach Second Printing 1949, S. 217.

37 Bereits G. Nottebohm und H. Deiters glaubten, der Satz sei zunächst für op. 132 bestimmt gewesen. Hierzu vergleiche die Polemik A. Scherings in dessen Arbeit: Beethoven in neuer Deutung, Leipzig 1934, S. 52, Anmerkung 1.

38 M. Carner, Artikel Walzer, in: MGG XIV', 1968, S. 228. 
Satz V (Più allegro) hervor, beide Male nicht als Gattungstypus bezeichnet, aber umsomehr spürbar. Merkwürdig, daß die (wohl auch im zweiten Fall »attaca«) anschließenden Sätze nicht eigentlich ein Arioso vertreten. Doch ist ihnen eine zentrierende, kreisende Melodik eigen, die wir bereits als Altersspezifikum beschrieben. Wie das genannte Ricercare, so ist auch das Rezitativ eine archaische Art, dem Hauptsatz ein »Vorfeld" beizugeben. Allen Fällen gemeinsam ist die spärliche, aufsteigend-tastende Einfädelung des Rezitativs. Das Maximum in der Mitte zeigt eine weitausladende Oberstimmen-Diminution. Die Endung enthält - abgesehen von der üblichen und atypischen Halbschlußbewegung - das fallende Sekundintervall scharf beleuchtet (op. $131 \mathrm{Baß,} \mathrm{op.132} \mathrm{Sopran).} \mathrm{In} \mathrm{beiden} \mathrm{Fällen} \mathrm{wird} \mathrm{aus}$ dieser engräumigen Halbkadenz das gefesselte Motivbild des Folgesatzes gewonnen. In der langen Reihe Beethovenscher Instrumental-Rezitative sehen wir hier einen neuen Standort: der frühe Meister liebte rezitativische Einschübe im Unisono eher als Kontrastmittel emotionaler Prägung gegenüber raschen Bewegungsreihen (zum Beispiel op. 31, Nr.2, Satz I, »largo, con espressione e semplice«). Später schritt Beethoven zu weitgeschwungenen Rezitativen fort, die auch auf Bebungseffekte nicht verzichten (op. 110, "una corda«, "tutte le corde«). Diese Entwicklung dürfte sich mit dem Übergang decken, den v. Fischer ${ }^{39}$ in dem Weg von »bewußt thematischer Prägung " zu »entwicklungsmotivischen Bildungen" richtig erkannt hat, eine schon von Riemann ${ }^{40}$ beobachtete Tendenz. In op. 131 und 132 ist hingegen als letzte Position die melodische Flexibilität verkümmert, der Klangstrom wird teils durch heftiges Tremolo beeinträchtigt, teils durch vorzeitiges Abbrechen fragwürdig gemacht. Ein wild-unversöhnlicher, gereizter Deklamationsstil eröffnet sich, bei dem die - als allgemeines "Sprachgut " untypische - "smorzando«-Endung fast nebensächlich wirkt. Mit diesem Merkmal hängt die Neigung zu "geschuppten" »Dauerrhythmen zusammen, wie sie in der Quartettfuge am eindrücklichsten geboten ist. Die "Alterswildheit", wie sie der Kunsthistoriker A. E. Brinckmann formulierte, wäre ein gewagter Vergleich, aber unbestritten bleibt die kurzschrittigrhythmische Dauer-Reihung neben dem anderen Extrem, der beschriebenen Dehnung melodischer Ganzheiten bis an die Grenze einheitlicher Wahrnehmung. Beide Merkmale treffen sich umgekehrt wieder mit der Eigenart, am Satzhöhepunkt neue Motive einzustreuen, was neben der genannten Rondo- und Couplet-Technik auch zeigt, daß Beethoven eine »streng thematische Gebundenheit « ${ }^{41}$ verläßt. Insofern dient auch der rezitativische Einschub einer improvisatorischen Anlage im Ganzen.

${ }^{39} \mathrm{~K}$. v. Fischer, Die Beziehungen von Form und Motiv in Beethovens Instrumentalwerken, 2. vermehrte Auflage, Baden-Baden 1972 (= Collection d'études musicologiques ..., Band XXX), S. 245.

$40 \mathrm{H}$. Riemann, Beethovens Streichquartette (= Schlesinger'sche Musik-Bibliothek, Meisterführer XII), Berlin 1903, S. $134 \mathrm{f}$.

${ }_{41}$ So richtig K. v. Fischer, a. a. O., S. 175. 
6. Die »optische« Priorität gegenüber der »klanglichen« ist unleugbar. Motivumkehrungen häufen sich. Breitgestaffelte Rückleitungs-Bewegungen, wobei die Außenstimmen sich langsam nähern, haben eher optische Relevanz, zumal sie nicht selten harte Durchgangs-Dissonanzen in Kauf nehmen (Quartettfuge, Takt 326-351 etc.). Hierzu rechnet auch die spiegelbildhafte Führung der Außenstimmen im engeren Raum (op. 130, Satz I, Takt 138 etc., op. 131, Satz IV, Takt 94 ff., 101 ff., 141 ff.), die Technik findet sich im letzten Klavierwerk vorbereitet (op. 109, Finalsatz, Variation 4, Schluß). Beethovens "Abstinenz" vom vordergründig-Klanglichen und seine Hinwendung zu "graphischen", zeichnerischen Wirkungen sollte ebenfalls nicht im hermeneutischspätromantischen Sinn als ein tragisches Lebensgefühl oder als eine Regression des alternden Musikers verstanden werden, sie erfahren auch mit der Taubheit keine ausreichende Begründung. Vielmehr liegt auch hier ein spezifisch musikalisches Merkmal der Reifung vor: Verhärtung zu Motivblöcken (op. 135 »Der schwer gefaßte Entschluß«) und die Tendenz, harte Ostinati in den Satz einzupflanzen. Das sind außeremotionale Merkmale, von äußerer Klangvision sich befreiend. Der selaboratio" ist deutlich (gegenüber einer »inventio«) der Vorrang gegeben. Die optische Übereinstimmung erlangt Vorrechte: man vergleiche in op.132, Satz IV "Alla marcia", Takt 8 (Ende der ersten Melodiephrase) mit dem Beginn des folgenden Rezitativs (Più Allegro). Die Identität zeigt sich in der Klangarmut und in der optischen Úbereinstimmung, obschon emotional zwei grundverschiedene Prozesse ablaufen (derbes Herausschleudern einer marschähnlichen Phrasenendung - tastender Anlauf zu einem dramatischen Sprechgesang).

7. Letztlich sei bemerkt, daß gegenüber Beethovens pianistischem Spätwerk der Quartettsatz auf weit ausschweifende Fiorituren und Diminution in der Oberstimme verzichtet (man halte den langsamen Satz der Hammerklaviersonate oder den Mittelteil der Sonate op.111, Satz II entgegen). Das kann nicht nur an der Eigenständigkeit des Quartettsatzes liegen. Vielmehr dürfte Beethoven zuletzt bewußt die in Höhenlagen des Klangs schweifende Umspielung abgebaut haben, was sich besonders auf die Progression der Glieder einer Variation ausgewirkt hat. In op. 132, Satz III, Takt 144 ff. wird nur noch ein einziges Mal die vom späten Klavierwerk her vertraute rasche Triolierung (oder Sextolen) verlangt, die ohnehin wegen der beabsichtigten Themenkontraste (»Neue Kraft fühlend«) kaum zu Tragen kommt.

Beethovens letzte Streichquartette sind eine Grenzsituation des Künstlerischen, die sich der im Schrifttum so oft unternommenen Idealisierung zu widersetzen scheint, ebenso wie diese Werkgruppe von den frühromantischen "Beethovenern" kaum recht verstanden wurde und im umgekehrten Sinn als »Gelehrsamkeit« unzugänglich blieb. Gewiß haben wir es mit einem seltenen Fall einer »Stilvollendung " zu tun, definiert als optimalen Grad einer Ausdruckssättigung, Einheit, Durchbildung im Einzelnen, als letztes Glied nach 
vorausgegangener artistischer Vervollkommnung, äußerer Sprachmeisterschaft, routinehafter Materialbeherrschung. Dabei liegt es nicht an der »Eleganz« der Lösung. Die Kongruenz der Teile ist oft bedenklich verschleiert und es werden »Umwege» beschritten. Es ist auch nicht nur die oft bemerkte Vergeistigung des Vitalen: der Begriffsdualismus »In der Jugend herrscht Anschauung, im Alter das Denken" (Schopenhauer), die Konfrontierung einer »funktionalen Befriedigung " zur »systematischen Aufgabe-Erfüllung ist vielleicht garnicht das bezeichnende Merkmal. Der späte Beethoven versinnlicht einen durchaus komplexen Sonderstatus, der auch von zeitstilistischen Prozessen überlagert ist. Sicher aber war das historische Selbstverständnis des »alten « Beethoven in eine Krise eingetreten, die auch seine unmittelbare Existenz, das Bewußtsein seiner Gegenwart nicht unberührt ließ. Vielleicht war es jene "Grenzsituation", die der alte Friedrich Hebbel so eindringlich formuliert hat: "Es ist, als ob die Welt in ihrem tiefsten Grunde aufgewühlt, die Form verändert. Das Vergangene ringt aus dem Grabe und das Künftige drängt zur Geburt, das Gegenwärt'ge aber setzt sich zur Wehre."

\section{POVZETEK}

Beethovnovi pozni godalni kvarteti op. 127 Es-dur (1822-1825), op. 130 B-dur (1825-26), op. 131 cis-mol (1825-26), op. 132 a-mol (1824-25), op. 135 F-dur (1825) in fuga v B-duru op. 133 (1825) so zaključene skupine in vzoren primer za pojem pozne ustvarjalnosti. Njihovo enovitost potrjuje določena oddaljenost od vsega, kar je napisal Beethoven poprej na področju simfonične in klavirske glasbe, kakor tudi od Missae solemnis. Pri poznem Beethovnu zbudi nasploh pozornost dejstvo, da se skladatelj skoro dosledno izogiba oznake "scherzo« in docela eliminira starejši tip menueta. Radikalna poenostavitev in zožitev scherzozne teme, ki se najavlja že v klavirski sonati op. 106 in stopnjuje v sonati op.110, se v poznih godalnih kvartetih še nadaljuje. Tako je tu za stavke, ki imajo funkcijo scherza, karakteristično, da je njih tematika kratkodahna in sunkovita. Razen tega nastopa sorazmerno pogosto togo vodenje glasov v unisonu, medtem ko imajo trilčki vse bolj funkcijo, da vzbujajo pojem grobosti. V poznih godalnih kvartetih tudi ni prezreti oslabitve, razredčenja specifične zvočnosti, za kar govori že samo dejstvo, da se Beethoven omejuje na štiriglasni stavek homogenih instrumentov. V tej zvezi je očitno skrajno visoko vodenje obeh violin, ki zvenita skoro kot piccolo flavta in pojavljanje statičnega, brnečega zvočnega kompleksa, ki včasih ob tesnem obigravanju v sekundah dejansko že kar "stoji«. Vsepovsod prisotna težnja za oslabitev zvočnosti seveda ne izključuje na posameznih mestih močno zgostitev in kopičenje zvoka, kar pa je razumeti predvsem kot izjemo od osnovnega pravila. Vsekakor svojstveni pečat dajejo poznim kvartetom svečano vzvišene teme, ki imajo svoj vzor $\mathrm{v}$ vokalni polifoniji 16 . stoletja in jih tedaj najdemo tudi $\mathrm{v}$ instrumentalnih ricercarih. Najbolj nazoren primer takšnega tipa teme dobimo v 1. stavku kvarteta op. 131. Gre za lebdečo temo, zasnovano v stile antico, pri kateri pogrešamo tisti krepko in jasno izoblikovani začetek, ki smo ga vajeni pri Beethovnu srednjega ustvarjalnega obdobja. Tu čutimo nekakšno »detipizacijo« in "relativizacijo« poteka teme, ki razločno na- 
sprotuje poprej Beethovnu tako lastni praksi "tematskega dela« in razodeva težnjo za "splošnim", za "razosebljenjem", težnjo, ki je osnova tudi drugemu delu Goethejevega Fausta. Popolno nasprotje te vrste tem so teme $\mathrm{z}$ izredno široko razprtimi intervalskimi postopi, za kar je navesti kot eminenten primer začetek kvarteta op. 132 in fugo op. 133. Nove so tudi pozibavajoče se, sproščene teme, ki se približujejo plesu. Tu je opozoriti predvsem na 4. stavek kvarteta op. 130 (Alla danza tedesca), pri čemer ne smemo pozabiti, da je bil v času nastanka te kompozicije že razširjen dunajski hitri valček. Nadalje je za stil poznih kvartetov, kot kažeta 3. stavek op. 131 (Allegro moderato, Più vivace) in 5. stavek op. 132 (Più allegro) karakteristična uporaba recitativa. Ta recitativ je podobno kot vokalna vzvišena tema arhaičnega značaja in se loči od recitativa v prejšnjih Beethovnovih instrumentalnih delih. Gre za divje vzburjeni, nespravljivi deklamacijski stil, kjer je melodična prožnost zakrnela in silovit tremolo zavira zvočni tok. Neutajljiva je tudi »optična« prioriteta nasproti "zvočni«. Inverzije motivov se kopičijo. Široko zasnovani prehodi $\mathrm{k}$ reprizi imajo $\mathrm{v}$ marsičem optični pomen, še posebno, ker se pojavljajo trde prehodne disonance. To odpovedovanje zvočnosti in nagibanje $h$ grafičnim efektom pa nima svoj vzrok $v$ skladateljevi osebni nesreči in gluhosti, ampak je kot sicer v pozni Beethovnovi ustvarjalnosti specifična muzikalna značilnost zorenja. Končno je še opozoriti, da se Beethoven za razliko od poslednjih sonat $\mathrm{v}$ poznih kvartetih odreka široko razpredenim fiorituram in diminucijam $\mathrm{v}$ zgornjem glasu. 